\title{
Simultaneous Photometric and Spectroscopic Observations of the unusual Variable F-type stars HD 224638 and HD 224945.
}

\author{
L. Mantegazza ${ }^{1}$, E. Poretti ${ }^{2}$, M. Bossi ${ }^{2}$ and F.M. Zerbi ${ }^{1}$ \\ ${ }^{1}$ Dipartimento di Fisica Nucleare e Teorica, Università di Pavia, Italy \\ 2 Osservatorio Astronomico di Brera, Italy
}

\section{Introduction}

The stars HD 224638 and HD 224945 belong to the new class of F0 V spectral type stars which show an unusual kind of variability for that region of the HR diagram (Mantegazza et al. 1993).

The variability of these two stars has been discovered and studied by Mantegazza, Poretti \& Zerbi (1994). They show small amplitude light variations with characteristic time scales of the order of one day. The light curves are not periodic and it is difficult to satisfactorily fit them even with several periodic terms.

In order to explain our $1991 B$ colour observations we tentatively suggested that in both stars two close periodic terms were present with a characteristic frequency of about $0.8 \mathrm{c} / \mathrm{d}$, and that these terms had a double wave shape for HD 224638 and a triple wave one for HD 224945.

Three high resolution spectrograms of HD 224638 also showed that probably there were line profile variations.

At the moment two competitive models are the most plausible in order to explain these variations: non-radial $g$-mode pulsations or spots carried across the visible stellar disk by rotation.

\section{New observations}

In order to better understand the phenomenology of these stars we performed simultaneous $B, V$ (13 nights, Oct. 23 - Nov. 6, 1994) and high resolution spectroscopic (5 nights, Oct. $24-29,1994)$ observations with the $50 \mathrm{~cm}$ and CAT telescopes respectively at $\mathrm{La}$ Silla (ESO). The spectrograms cover the region between 4490 and $4525 \AA$ with a resolution of 60000 and have a mean $\mathrm{S} / \mathrm{N}$ ratio at a continuum level of about 250 .

A preliminary data analysis allows us to establish the following facts:

- The light curves have a frequency content very similar to that of previous observations in 1991. For example for HD 224638 we find 5 terms with frequencies of $1.66,1.37,1.56,0.96$ and $1.69 \mathrm{c} / \mathrm{d}$ in the 1994 data and of $1.62,1.37,1.70,0.89$ and $0.81 \mathrm{c} / \mathrm{d}$ in the 1991 data.

- The frequency analysis of colour index data indicates that the colour index varies approximately in phase with the light. 


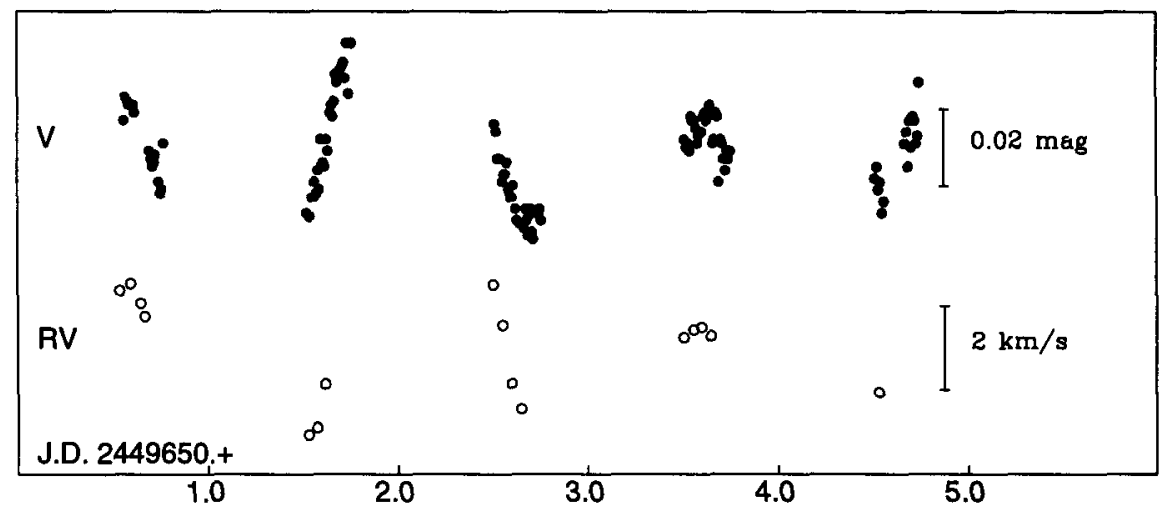

Figure 1. V and radial velocity curves of HD 224638

- There are large variations in the shapes of line profiles, which are also detectable in the course of the same observing night.

- The frequency analysis of the time-series of the first 5 moments of the line profiles (Balona 1986), indicates that they have approximately the same frequency content as does the photometric data.

- The full amplitudes of the radial velocity variations ( $m 1$ moment) are of about $3.5 \mathrm{~km} / \mathrm{s}$ for both stars. Fig. 1 shows that for HD 224638 these variations are in phase with the light curves. This behaviour is the opposite to that observed in radial and non-radial $p$-mode pulsators in the instability strip.

\section{Conclusion}

The observed light, colour and line profile variations could in principle be explained both by the pulsation and the spot model. However the spot model can hardly explain both the observed amplitudes in radial velocities and the presence of several periodicities stable on a baseline of a few years. In order to definitely confirm this last point it would be of paramount importance to perform a multi-site campaign on these stars, due to the difficulty of deconvolving unambiguously from the spectral window time-series with periods of the order of 1 day.

\section{References}

Mantegazza, L., Poretti, E., Antonello, E., \& Zerbi, F.M. 1993, in: Inside the Stars, W.W. Weiss \& A. Baglin, ASP Conf. Ser. 40, 733

Mantegazza, L., Poretti, E., \& Zerbi, F.M. 1994, MNRAS, 270, 439

Balona, L. 1986, MNRAS, 219, 111 